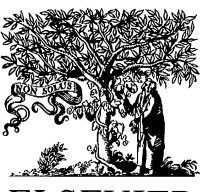

ELSEVIER

\title{
FIR $H^{\infty}$ equalization
}

\author{
A.T. Erdogan ${ }^{\mathrm{a}, *}$, B. Hassibi ${ }^{\mathrm{b}}$, T. Kailath ${ }^{\mathrm{c}}$ \\ ${ }^{a}$ Virata Corporation, Cupertino, CA, USA \\ ${ }^{\mathrm{b}}$ Lucent Technologies, NJ, USA
}

'Information Systems Laboratory, Electrical Engineering, Stanford University, 844 Cedro Way, Stanford, CA 94305, USA

Received 9 March 2000; received in revised form 4 October 2000

\begin{abstract}
We approach finite impulse response (FIR) equalization problem from an $H^{\infty}$ perspective. First, we formulate the calculation of the optimal $H^{\infty}$ performance for a given equalization setting as a semidefinite programming (SDP) problem. $H^{\infty}$ criterion provides a set of FIR equalizers with different optimality properties. Among these, we formulate the calculation of risk-sensitive or minimum entropy FIR filter as the constrained analytic centring problem and mixed $H^{2} / H^{\infty}$ problem as another SDP. We provide examples to illustrate the procedures we described. (C) 2001 Elsevier Science B.V. All rights reserved.
\end{abstract}

Keywords: FIR equalization; H-infinity; Risk-sensitive criterion; Max-det programming

\section{Introduction}

In Refs. [9,3,4], $H^{\infty}$ criterion has been applied to the linear and decision feedback equalization problems with the belief that the resulting $H^{\infty}$ equalizers will be more robust with respect to model uncertanities and the lack of statistical knowledge of the exogenous signals. With this new approach, various new insights are obtained related to the equalization problem, like the roles of minimum phase property and delay. Besides this approach provides a good basis for the future work for the design of robust equalizers dealing with modeling errors. In the work of above-cited references, there is no con-

\footnotetext{
* Correspondence address. Information Systems Laboratory, Electrical Engineering, Stanford University, 844 Cedro Way, Stanford, CA 94305, USA. Tel.: + 1-650-320-9607.

E-mail address: alper@stanfordalumni.org (A.T. Erdogan).
}

straint on the length of the equalizers. The resulting equalizers may therefore be infinite impulse response (IIR) filters. In this article, we look at the FIR equalization problem from an $H^{\infty}$ perspective.

There are various reasons for the preference of FIR equalizers over the IIR filters. First of all, FIR equalizers have very low complexity and are easy to implement. Furthermore, the IIR filters may suffer from limit cycles caused by the finite precision implementation in real systems and the recursive structure of the IIR filter. Finally, the majority of the adaptive equalization approaches make the FIR assumption and that generally provides the property that the cost function (either stochastic or deterministic) is a convex function of the FIR equalizer coefficients and has therefore, a single globally optimal solution.

Within the FIR $H^{\infty}$ equalization framework, the first problem that we are going to address is the 
calculation of the optimal achievable $H^{\infty}$ performance ( $\gamma$ value) for the FIR equalizers. We use the state-space representation, which is presented in the next section, in conjunction with the $\mathrm{Ka}$ lman-Yakubovich-Popov (KYP) Lemma [6] to pose this problem as a convex semidefinite programming (SDP) problem. Then we change our focus to the calculation of FIR $H^{\infty}$ equalizer coefficients. First, as a major result of this paper, we formulate the FIR risk sensitive equalization problem as a constrained analytic centering problem, which is another type of convex problem (MAXDET) with linear matrix inequality (LMI) constraints. Next, we investigate the mixed $H^{2} / H^{\infty}$ FIR equalization. In the FIR case, since the equalizer is constrained to use only a finite number of observation samples, the solution is expected to be far from the smoothing solution. Therefore, the $H^{2}$ and $H^{\infty}$ solutions are different in general. We later show that the mixed $H^{2} / H^{\infty}$ FIR equalizer, that is, the $H^{\infty}$ optimal FIR equalizer with the least $H^{2}$ cost, can be calculated using SDP.

\section{State-space description for the error transfer function}

Fig. 1 illustrates the general structure of the error transfer function $T_{K}$ corresponding to the equalization problem. Here $\left\{b_{i}\right\}$ is the transmitted digital information sequence, $H(z)$ is the discrete equivalent of the linear time-invariant communication channel and $\left\{v_{i}\right\}$ is the unknown noise corrupting

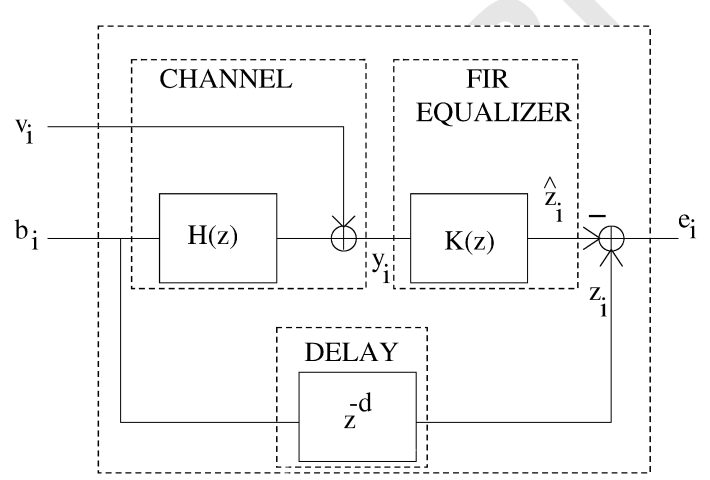

Fig. 1. Error transfer function. the observations. FIR equalization problem is to design FIR filter $K(z)$ to minimize the error $\left\{e_{i}\right\}$ between the equalizer output and the delayed version of the desired transmitted sequence with respect to a chosen criterion. In this section, we derive a state-space representation for the error transfer function $T_{K}$, which is the mapping from input disturbances $\left\{b_{i}\right\}$ and $\left\{v_{i}\right\}$ to the equalization error sequence $\left\{e_{i}\right\}$. In Section 3, we use this state-space description in conjunction with the KYP Lemma to calculate the optimal $\gamma$ value for the $H^{\infty}$ optimal FIR equalizers.

We first begin by listing state-space descriptions for the components of the error transfer function: the channel $H(z)$, equalizer $K(z)$ and the delay $L(z)=z^{-d} I$.

The channel $H(z)$, in combination with the noise at the output, is assumed to have the following state-space structure:

$$
\begin{aligned}
& \beta_{i+1}=F_{c} \beta_{i}+\underbrace{\left[\begin{array}{ll}
g_{c} & 0
\end{array}\right]}_{G_{c}}\left[\begin{array}{l}
b_{i} \\
v_{i}
\end{array}\right], \\
& y_{i}=H_{c} \beta_{i}+\underbrace{\left[\begin{array}{ll}
h_{0} & I
\end{array}\right]}_{C_{0}}\left[\begin{array}{l}
b_{i} \\
v_{i}
\end{array}\right] .
\end{aligned}
$$

The state structure for the delay operator $L(z)=z^{-d} I$ is

$$
\begin{aligned}
& \zeta_{i+1}=\underbrace{\left[\begin{array}{cc}
0 & 0 \\
I_{(d-1) \cdot M} & 0
\end{array}\right]}_{F_{d}} \zeta_{i}+\underbrace{\left[\begin{array}{cc}
I & 0 \\
0 & 0
\end{array}\right]}_{G_{d}}\left[\begin{array}{c}
b_{i} \\
v_{i}
\end{array}\right], \\
& z_{i}=\underbrace{\left[\begin{array}{ll}
0 & I
\end{array}\right]}_{\boldsymbol{H}_{d}} \zeta_{i},
\end{aligned}
$$

when $d>0$, and

$z_{i}=b_{i}$

when $d=0$.

Finally, the state-space structure for the FIR equalizer $K(z)=k_{0}+k_{1} z^{-1}+\cdots+k_{R-1} z^{-(R-1)}$ with order $R-1$ has the following state-space structure:

$$
\begin{aligned}
& \xi_{i+1}=\underbrace{\left[\begin{array}{cc}
0 & 0 \\
I_{(R-2) \cdot N} & 0
\end{array}\right]}_{F_{e}} \xi_{i}+\underbrace{\left[\begin{array}{c}
I \\
0_{(R-2) \cdot N}
\end{array}\right]}_{G_{e}} y_{i}, \\
& \hat{z}_{i}=[\underbrace{k_{1} \ldots k_{R-1}}_{H_{e}}] \xi_{i}+k_{0} y_{i} .
\end{aligned}
$$


Given these, we can obtain the state-space structure for the error transfer function that maps input disturbances

$$
\left[\begin{array}{l}
b_{i} \\
v_{i}
\end{array}\right]
$$

to error $e_{i}=z_{i}-\hat{z}_{i}$ :

$$
x_{i+1}=\underbrace{\left[\begin{array}{ccc}
F_{c} & 0 & 0 \\
0 & F_{d} & 0 \\
G_{e} H_{c} & 0 & F_{e}
\end{array}\right]}_{F} x_{i}+\underbrace{\left[\begin{array}{c}
G_{c} \\
G_{d} \\
G_{e} C_{0}
\end{array}\right]}_{G}\left[\begin{array}{c}
b_{i} \\
v_{i}
\end{array}\right],
$$

$$
\begin{aligned}
y_{i}= & \underbrace{\left[\begin{array}{lll}
-k_{0} H_{c} & H_{d} & -H_{e}
\end{array}\right]}_{H} \\
& \times x_{i}+(\underbrace{-k_{0} C_{0}}_{D})\left[\begin{array}{l}
b_{i} \\
v_{i}
\end{array}\right],
\end{aligned}
$$

when $d>0$, and

$$
\begin{aligned}
& x_{i+1}= {\left[\begin{array}{cc}
F_{c} & 0 \\
G_{e} H_{c} & F_{e}
\end{array}\right] x_{i}+\left[\begin{array}{c}
G_{c} \\
G_{e} C_{0}
\end{array}\right]\left[\begin{array}{l}
b_{i} \\
v_{i}
\end{array}\right], } \\
& y_{i}=\left[\begin{array}{ll}
-k_{0} H_{c} & -H_{e}
\end{array}\right] x_{i} \\
&+\left(\left[\begin{array}{ll}
I & 0
\end{array}\right]-k_{0} C_{0}\right)\left[\begin{array}{l}
b_{i} \\
v_{i}
\end{array}\right],
\end{aligned}
$$

when $d=0$. In the above state-space formulation, the only terms that depend on the equalizer coefficients $k_{0}, \ldots, k_{R-1}$ are $H$ and $k_{0} C_{0}$. Given this state-space model, we can write the error transfer function as

$T_{K}(z)=H(z I-F)^{-1} G+D$.

Therefore, in this representation, only $H$ and $D$ are linear functions of the equalizer coefficients.

\section{Calculation of the optimal achievable $H^{\infty}$ performance for FIR equalizers}

In Refs. [3,4], the optimal $H^{\infty}$ performance level for linear and decision feedback IIR equalizers are studied. One subject of interest is to investigate how the performance of the equalizers are affected by the FIR constraint. In this section, we provide the calculation of the optimal achievable level for FIR equalizers using convex optimization techniques.

We start by stating the $H^{\infty}$ FIR equalization problem as

$$
\begin{aligned}
\inf _{K(z)} & =k_{0}+k_{1} z^{-1}+\cdots+k_{R-1} z^{-(R-1)} \\
& \max _{\omega} \sigma_{\max }\left(T_{K}\left(\mathrm{e}^{\mathrm{j} \omega}\right)\right) \\
& =\gamma_{\text {opt,fir }},
\end{aligned}
$$

where $\sigma_{\max }()$ is the maximum singular value of its argument. In other words, FIR $H^{\infty}$ equalization problem is to find a finite-dimensional matrix $\left[k_{0} \ldots k_{R-1}\right]$ such that maximum gain of the error transfer function $T_{K}\left(\mathrm{e}^{\mathrm{j} \omega}\right)$ is minimized over all possible frequencies. Therefore, for a given $\gamma \geqslant \gamma_{\text {opt,fir }}$, for $K(z)=k_{0}+k_{1} z^{-1}+\cdots+k_{R-1} z^{-(R-1)}$ to be an optimal $\gamma$-level $H^{\infty}$ FIR filter, it should satisfy

$$
T_{K}\left(\mathrm{e}^{\mathrm{j} \omega}\right)^{*} T_{K}\left(\mathrm{e}^{\mathrm{j} \omega}\right) \leqslant \gamma^{2} I \quad \forall \omega \in[0,2 \pi) .
$$

Equivalently, if we can find a $\gamma$ such that Eq. (14) is satisfied, then $\gamma \geqslant \gamma_{\text {opt }}$, i.e., $\gamma$ is achievable. Using the state-space formulation we defined in the previous section, we can give an equivalent condition to the frequency domain condition of Eq. (14). The basic tool we employ for this purpose is the KYP lemma which we state here without proof and refer to (6) for the proof:

Lemma 1 (KYP Lemma). Consider the observable pair $\{A, C\}$. Then the following two statements are equivalent:

(1) $S_{y}(z) \geqslant 0$ for all $z=\mathrm{e}^{\mathrm{j} \omega} \notin \lambda(F)$ where

$$
\begin{aligned}
S_{y}(z)= & {\left[C(z I-A)^{-1} \quad I\right]\left[\begin{array}{cc}
Q & S \\
S^{*} & R
\end{array}\right] } \\
& \times\left[\begin{array}{c}
\left(z^{-1} I-A^{*}\right)^{-1} C^{*} \\
I
\end{array}\right] .
\end{aligned}
$$

(2) There exists a Hermitian $Z$ such that

$$
\left[\begin{array}{cc}
Q-Z+A Z A^{*} & S+A Z C^{*} \\
S^{*}+C Z A^{*} & R+C Z C^{*}
\end{array}\right] \geqslant 0 .
$$

The KYP lemma converts the task of checking positivity of the frequency domain function for all frequencies to the task of finding a Hermitian matrix satisfying a linear matrix inequality (LMI).

In order to use the KYP lemma, we need to convert Eq. (14) into the form of Eq. (15). The first 
step in this process is to use the Schur complement method which is outlined in the following lemma:

Lemma 2. The following statements are equivalent:

(1) For $\gamma \geqslant 0$

$$
T_{K}^{*}\left(\mathrm{e}^{\mathrm{j} \omega}\right) T_{K}\left(\mathrm{e}^{\mathrm{j} \omega}\right) \leqslant \gamma^{2} I \quad \forall \omega \in[0,2 \pi) .
$$

(2)

$$
\left[\begin{array}{ll}
I & T_{K}\left(\mathrm{e}^{\mathrm{j} \omega}\right) \\
T_{K}^{*}\left(\mathrm{e}^{\mathrm{j} \omega}\right) & \gamma^{2} I
\end{array}\right] \geqslant 0 \quad \forall \omega \in[0,2 \pi) .
$$

Proof. The second statement is true if and only if $I$ and its Schur complement $\gamma^{2} I-T_{K}^{*}\left(\mathrm{e}^{\mathrm{j} \omega}\right) T_{K}\left(\mathrm{e}^{\mathrm{j} \omega}\right)$ are non-negative, which is the first statement.

In order to put the matrix in (18) to the form of $S_{y}(z)$ in (15), we perform the following steps:

$$
\begin{aligned}
& {\left[\begin{array}{cc}
I & T_{K}\left(\mathrm{e}^{\mathrm{j} \omega}\right) \\
T_{K}^{*}\left(\mathrm{e}^{\mathrm{j} \omega}\right) & \gamma^{2} I
\end{array}\right]} \\
& =\left[\begin{array}{cc}
I & H\left(\mathrm{e}^{\mathrm{j} \omega} I-F\right)^{-1} G+D \\
D^{*}+G^{*}\left(\mathrm{e}^{-\mathrm{j} \omega} I-F^{*}\right)^{-1} H^{*} & \gamma^{2} I
\end{array}\right] \\
& =\underbrace{\left[\begin{array}{c}
0 \\
G^{*}
\end{array}\right]}_{C}(\mathrm{e}^{-\mathrm{j} \omega} I-\underbrace{F^{*}}_{A})^{-1} \underbrace{\left[\begin{array}{cc}
H^{*} & 0
\end{array}\right]}_{S} \\
& +\left[\begin{array}{c}
H \\
0
\end{array}\right]\left(\mathrm{e}^{\mathrm{j} \omega} I-F\right)^{-1}\left[\begin{array}{ll}
0 & G
\end{array}\right]+\left[\begin{array}{ll}
I & D \\
D^{*} & \gamma^{2} I
\end{array}\right] \\
& =\left[C\left(\mathrm{e}^{-\mathrm{j} \omega} I-A\right)^{-1} \quad I\right]\left[\begin{array}{c}
0 \\
S^{*}[\underbrace{S}_{R} \begin{array}{cc}
I & D \\
D^{*} & \gamma^{2} I
\end{array}]
\end{array}\right] \\
& {\left[\begin{array}{c}
\left(\mathrm{e}^{\mathrm{j} \omega} I-A^{*}\right)^{-1} C^{*} \\
I
\end{array}\right] .}
\end{aligned}
$$

We can use the KYP lemma to conclude that if $\gamma \geqslant \gamma_{\text {opt,fir }}$ then there exists a vector $\left[\begin{array}{lll}k_{0} & \ldots & k_{R-1}\end{array}\right]$ and a Hermitian matrix $Z$ such that

$$
\left[\begin{array}{cc}
-Z+A Z A^{*} & S+A Z C^{*} \\
S^{*}+C Z A^{*} & R+C Z C^{*}
\end{array}\right] \geqslant 0 .
$$

Note that (23) is an LMI in $k=\left[\begin{array}{lll}k_{0} & \ldots & k_{R-1}\end{array}\right]^{\mathrm{T}}$, $Z$ and $\gamma$. Therefore, we can define the calculation of the optimal value of $\gamma_{\text {opt,fir }}$ as a semi-definite programming (SDP) problem:

minimize $\gamma$

subject to $F(k, Z, \gamma) \geqslant 0$,

where

$$
F(k, Z, \gamma)=\left[\begin{array}{cc}
-Z+A Z A^{*} & S+A Z C^{*} \\
S^{*}+C Z A^{*} & R+C Z C^{*}
\end{array}\right] .
$$

This solution yields both the optimal value of $\gamma_{\text {opt,fir }}$ and a feasible $H^{\infty}$ optimal FIR filter $\left[\begin{array}{llll}k_{0} & \ldots & k_{R-1}\end{array}\right]^{\mathrm{T}}$.

\section{Examples of $\gamma$ calculation}

In the previous section, we formulated the calculation of the optimal value of $\gamma$ as an SDP problem. In this section, we will illustrate the results obtained through use of this SDP formulation. Here we should note that the delay $d$ in each case is chosen as greater than the number of non-minimum phase zeros of the channel such that $\gamma<1$ [3].

In the first example, we choose the channel as $H(z)=1+0.33562 z^{-1}+4.6276 z^{-2}-0.14487 z^{-3}$ $+1.6837 z^{-4}$. This channel has two non-minimum phase zeros and two minimum phase zeros as shown in Fig. 2. When the equalization delay is chosen as $d=2$, the behavior of the optimal $\gamma$ as a function of equalizer length is also shown in the same figure. The solid line at the bottom of the right hand figure is the $\gamma_{\text {opt }}$ for the unconstrained smoothing filter. As can be seen from this figure, this value is almost achieved when the FIR equalizer length is greater than or equal to 7 .

In the second example, we consider $H(z)$ is equal to $1+1.9326 z^{-1}-1.2229 z^{-3}-2.493 z^{-3}+$ $2.1076 z^{-4}$. Similar to the previous example, this channel also has two non-minimum and two minimum phase zeros as shown in Fig. 3, and the delay is taken to be $d=2$. Since the zeros of the channel are closer to the unit circle in this case, the optimal $\gamma$ values are higher than in the previous example. In Fig. 3, the dash-dot line at the bottom of the righthand side figure represents the $\gamma_{\text {opt }}$ value of the unconstrained smoothing filter whereas the solid line represents the $\gamma$ level for the $H^{\infty}$-optimal IIR 

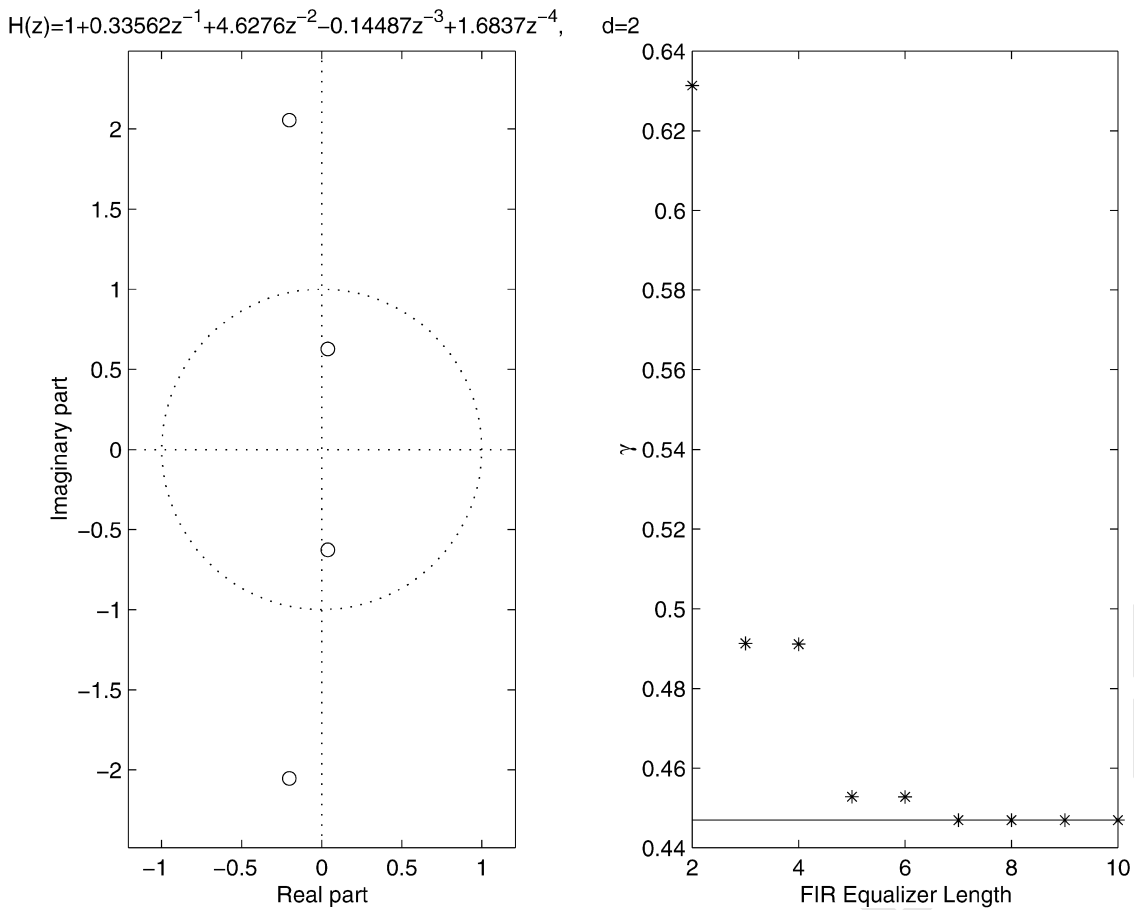

Fig. 2. Channel zero plot and the optimal value of $\gamma$ as a function of equalizer length for $H(z)=1+0.33562 z^{-1}+4.6276 z^{-2}$ $-0.14487 z^{-3}+1.6837 z^{-4}$.

equalizer with the same delay (i.e., $d=2$ ). As the equalizer length is increased, the optimal $\gamma$ value decreases and becomes closer to this level. However, there is still some offset even for an equalizer length of 10. Clearly, this offset should go to zero as the equalizer length is increased further towards $\infty$.

For the last example, we choose the channel as

$$
\begin{aligned}
H(z)= & 0.04-0.05 z^{-1}+0.07 z^{-2}-0.21 z^{-3} \\
& -0.5 z^{-4}+0.72 z^{-5}+0.36 z^{-6} \\
& +0.21 z^{-7}+0.03 z^{-8}+0.07 z^{-9}
\end{aligned}
$$

which is an example of a telephone channel [10], and which has five non-minimum phase zeros as shown in Fig. 4. The delay for this example is chosen as $d=6$. The solid line in Fig. 4 represents the $\gamma$ level for the smoothing equalizer and it is almost achieved by an FIR equalizer of length 5 .

\section{Risk-sensitive (minimum entropy) FIR equalization}

The set of $\gamma$-level $H^{\infty}$ optimal FIR filters where $\gamma \geqslant \gamma_{\text {opt,fir }}$ is a convex set which can be written as

$K_{\gamma}=\{k: \exists$ a hermitian $Z, F(k, Z, \gamma) \geqslant 0\}$.

All these filters in set $K_{\gamma}$ have different optimality properties with respect to different criteria. In applications, we desire the FIR equalizer to have some "average" optimality property besides being $H^{\infty}$ optimal. Our aim in this section is to come up with the FIR equalizer which is the member of $K_{\gamma}$ with the minimum risk-sensitive cost where the risk-sensitive cost function [14] is

$$
-\log \left(E\left(\exp \left(-\frac{2}{\gamma^{2}} \sum_{j=0}^{i} e_{j}^{*} e_{j}\right)\right)\right) .
$$

The resulting filter has the additional property of being the minimum entropy FIR filter $[5,14]$. In the 

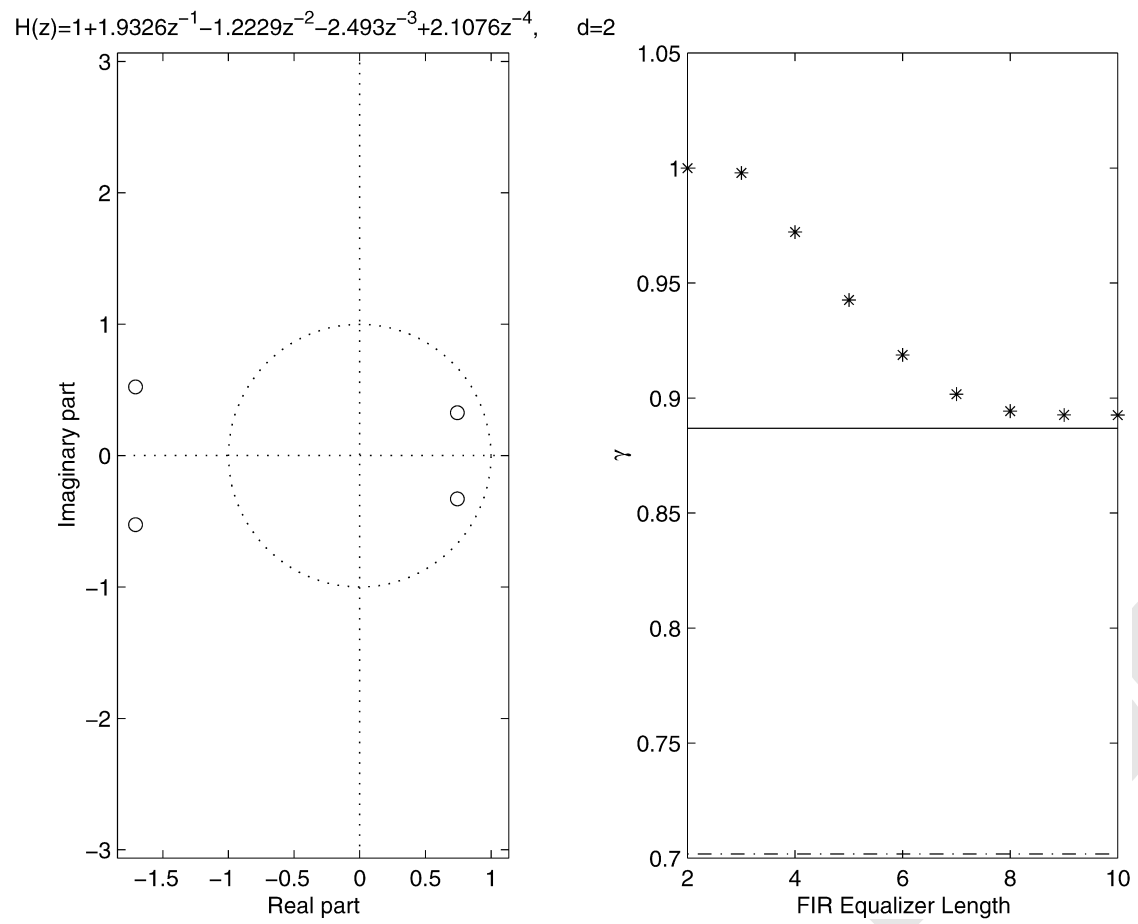

Fig. 3. The channel zero plot and the optimal value of $\gamma$ as a function of equalizer length for $H(z)=1+1.9326 z^{-1}-1.2229 z^{-3}$ $-2.493 z^{-3}+2.1076 z^{-4}$.

general $H^{\infty}$ setup, the central solution is the risksensitive equalizer, however, it is not necessarily FIR.

For the infinite horizon case, we can restate the FIR risk-sensitive equalization problem as

$$
\min _{k \in K_{\gamma}}-\int_{-\pi}^{\pi} \log \left(\operatorname{det}\left(\gamma^{2}-T_{K}\left(\mathrm{e}^{\mathrm{j} \omega}\right) T_{K}^{*}\left(\mathrm{e}^{\mathrm{j} \omega}\right)\right)\right) \mathrm{d} \omega .
$$

Note that this minimum entropy cost function is clearly a convex function of the equalizer coefficients. Furthermore, since the set $K_{\gamma}$ is a convex set, the problem in (30) is a convex optimization problem. In the rest of this section, we will convert the cost function involving an integral into a more compact expression containing the state-space variables for the error transfer function $T_{K}$. We first note that

$$
\begin{aligned}
& \operatorname{det}\left(\gamma^{2} I-T_{K}\left(\mathrm{e}^{\mathrm{j} \omega}\right) T_{K}^{*}\left(\mathrm{e}^{\mathrm{j} \omega}\right)\right) \\
& =\operatorname{det}(\underbrace{\left[\begin{array}{cc}
I & T_{K}^{*}\left(\mathrm{e}^{\mathrm{j} \omega}\right) \\
T_{K}\left(\mathrm{e}^{\mathrm{j} \omega}\right) & \gamma^{2} I
\end{array}\right]}_{S\left(\mathrm{e}^{\mathrm{j} \omega}\right)}) .
\end{aligned}
$$

Since over the set $K_{\gamma}, S\left(\mathrm{e}^{\mathrm{j} \omega}\right) \geqslant 0$, we can define

$S\left(\mathrm{e}^{\mathrm{j} \omega}\right)=\Delta_{S}\left(\mathrm{e}^{\mathrm{j} \omega}\right) R_{e} \Delta_{S}^{*}\left(\mathrm{e}^{\mathrm{j} \omega}\right), \quad \omega \in[0,2 \pi)$,

where $\Delta_{S}(z)$ is a monic, causal and causally invertible matrix and $R_{e}=R+C P C^{*}$. Here $P$ is the solution of the Riccati equation

$$
\begin{aligned}
- & P+A P A^{*} \\
& -\left(A P C^{*}+S\right)\left(R+C P C^{*}\right)^{-1}\left(A P C^{*}+S\right)^{*} \\
& =0 .
\end{aligned}
$$

The observability of $(A, C)$ implies the existence of $P(12)$. Since $\Delta_{S}$ is analytic for $|z| \geqslant 1$, it can be shown that

$$
\begin{aligned}
& \int_{-\pi}^{\pi} \log \left(\operatorname{det}\left(\gamma^{2}-T_{K}\left(\mathrm{e}^{\mathrm{j} \omega}\right) T_{K}^{*}\left(\mathrm{e}^{\mathrm{j} \omega}\right)\right)\right) \mathrm{d} \omega \\
& \quad=\log \left(\operatorname{det}\left(R_{e}\right)\right) .
\end{aligned}
$$

Therefore, we can rewrite the optimization problem of (30) as

$$
\min -\log \left(\operatorname{det}\left(R+C P C^{*}\right)\right)
$$




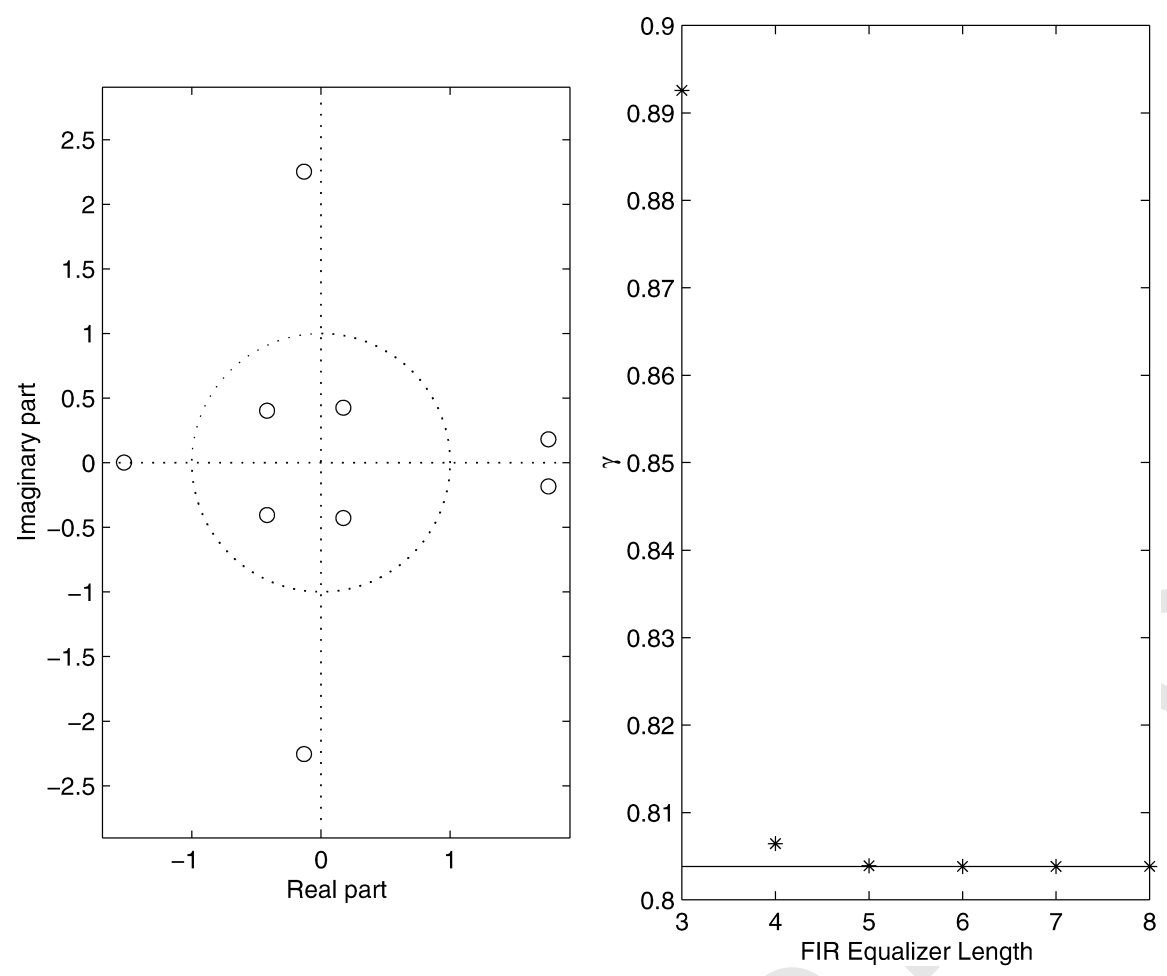

Fig. 4. Optimal value of $\gamma$ as a function of equalizer length for $H(z)$ given in Eq. (27).

s.t.

$$
\begin{aligned}
& -P+A P A^{*} \\
& -\left(A P C^{*}+S\right)\left(R+C P C^{*}\right)^{-1}\left(A P C^{*}+S\right)^{*}=0 .
\end{aligned}
$$

Although this formulation looks more desirable than (30), it contains a non-linear equality constraint. Our aim is to eliminate this non-linear constraint. For that purpose, we introduce the following convex optimization problem:

$\underset{k, Z}{\min }-\log \left(\operatorname{det}\left(R+C Z C^{*}\right)\right)$

$$
F(k, Z, \gamma) \geqslant 0 \text {. }
$$

The convex optimization problem in (36) is called a "constrained analytical centering" problem which is a special case of the more general MAXDET problem [15]. It involves a nonlinear convex barrier function as the cost function and the convex LMI constraints. It can be solved very efficiently using interior point methods [15]. If $Z_{\mathrm{o}}$ of the optimal solution $\left(k_{\mathrm{o}}, Z_{\mathrm{o}}\right)$ of the problem (36) satisfies the Riccati equation

$$
\begin{aligned}
-Z_{\mathrm{o}}+A Z_{\mathrm{o}} A^{*}- & \left(A Z_{\mathrm{o}} C^{*}+S_{\mathrm{o}}\right)\left(R_{\mathrm{o}}+C Z_{\mathrm{o}} C^{*}\right)^{-1} \\
& \times\left(A Z_{\mathrm{o}} C^{*}+S_{\mathrm{o}}\right)^{*}=0,
\end{aligned}
$$

where $R_{\mathrm{o}}$ and $S_{\mathrm{o}}$ are the values of $R$ and $S$ matrices at the optimal point, then it is easy to see that $k_{\mathrm{o}}$ will be the solution of the problem (35) and therefore (30).

In fact, this is generically the case which can be concluded via use of the following theorem [8]:

Theorem 1 (Maximal Hermitian solution). Consider the basic discrete Algebraic Riccati equation (DARE):

$$
\begin{aligned}
X= & F^{*} X F+Q \\
& -\left(S+X F H^{*}\right)^{*}\left(R+H X H^{*}\right)^{-1}\left(S+F X H^{*}\right)^{*}
\end{aligned}
$$


and the discrete Riccati inequality

$$
\begin{aligned}
- & X+F X F^{*}+Q \\
& -\left(S+F X H^{*}\right)\left(R+H X H^{*}\right)^{-1}\left(S+F X H^{*}\right)^{*} \\
& \geqslant 0 .
\end{aligned}
$$

Let $(F, H)$ be a detectable pair, $R$ be invertible and assume that there is a hermitian solution $\hat{X}$ of (39) for which $R+H \hat{X} H^{*}>0$. Then there exists a unique solution $X_{+}=X_{+}^{*}$ of (38) such that $R+$ $H X_{+} H^{*}>0$ and $X_{+} \geqslant X$ for all hermitian solutions of (38).

The above theorem directly implies that given $(A, C)$ in (37) is an observable and therefore a detectable pair, the maximality property of the Riccati equation solution implies the minimality of the $-\log \left(\operatorname{det}\left(R+C Z C^{*}\right)\right)$ and therefore $Z_{\mathrm{o}}$ should satisfy the Riccati equation (37).

We will now take an alternative route to prove this fact via the use of Karush-Kuhn-Tucker (KKT) optimality conditions [2] for the optimization problem (36). We begin by introducing the corresponding Lagrangian function as

$$
\begin{aligned}
& L(Z, k, W) \\
& \quad=-\log \left(\operatorname{det}\left(R+C Z C^{*}\right)\right)-\operatorname{Tr}(W F(k, Z, \gamma)),
\end{aligned}
$$

where $W \geqslant 0$ is the dual Lagrange matrix variable. Assuming strict feasibility and therefore the strong duality condition, the complementary slackness implies $[1,13]$ that at the optimal point $\left(k_{\mathrm{o}}, Z_{\mathrm{o}}, W_{\mathrm{o}}\right)$,

$W_{\mathrm{o}} F\left(k_{\mathrm{o}}, Z_{\mathrm{o}}, \gamma\right)=0$

$$
\begin{aligned}
& {\left[\begin{array}{ll}
W_{11, \mathrm{o}} & W_{12, \mathrm{o}} \\
W_{12, \mathrm{o}}^{*} & W_{22, \mathrm{o}}
\end{array}\right]} \\
& {\left[\begin{array}{cc}
-Z_{\mathrm{o}}+A Z_{\mathrm{o}} A^{*} & S_{\mathrm{o}}+A Z_{\mathrm{o}} C^{*} \\
S_{\mathrm{o}}^{*}+C Z_{\mathrm{o}} A^{*} & R_{\mathrm{o}}+C Z_{\mathrm{o}} C^{*}
\end{array}\right]=0,}
\end{aligned}
$$

which further implies,

$$
\begin{aligned}
W_{12, \mathrm{o}}= & -W_{11, \mathrm{o}}\left(S_{\mathrm{o}}+A Z_{\mathrm{o}} C^{*}\right)\left(R_{\mathrm{o}}+C Z_{\mathrm{o}} C^{*}\right)^{-1}, \\
W_{22, \mathrm{o}}= & \left(R_{\mathrm{o}}+C Z_{\mathrm{o}} C^{*}\right)^{-1}\left(S_{\mathrm{o}}+A Z_{\mathrm{o}} C^{*}\right)^{*} W_{11, \mathrm{o}} \\
& \times\left(S_{\mathrm{o}}+A Z_{\mathrm{o}} C^{*}\right)\left(R_{\mathrm{o}}+C Z_{\mathrm{o}} C^{*}\right)^{-1},
\end{aligned}
$$

$$
\begin{aligned}
0= & W_{11, \mathrm{o}}\left(-Z_{\mathrm{o}}+A Z_{\mathrm{o}} A^{*}-\left(A Z_{\mathrm{o}} C^{*}+S_{\mathrm{o}}\right)\right. \\
& \left.\times\left(R_{\mathrm{o}}+C Z_{\mathrm{o}} C^{*}\right)^{-1}\left(A Z_{\mathrm{o}} C^{*}+S_{\mathrm{o}}\right)^{*}\right) .
\end{aligned}
$$

Note that Eq. (45) implies that if $W_{11, \mathrm{o}}>0$, i.e., strictly positive, then Eq. (37) should hold. In order to check this condition, we use the first-order optimality condition of the Lagrangian function.

If we differentiate the Lagrangian function with respect to the matrix $Z$, we obtain

$$
\begin{aligned}
& \nabla_{Z} L(k, Z, W) \\
&=\nabla_{Z}\left(-\log \left(\operatorname{det}\left(R+C Z C^{*}\right)\right)\right) \\
& \quad-\nabla_{Z}\left(\operatorname{Tr}\left(W_{11}\left(-Z+A Z A^{*}\right)\right)\right) \\
& \quad-\nabla_{Z}\left(\operatorname{Tr}\left(W_{12}^{*}\left(A Z C^{*}+S\right)\right)\right) \\
& \quad-\nabla_{Z}\left(\operatorname{Tr}\left(W_{12}\left(A Z C^{*}+S\right)^{*}\right)\right) \\
&-\nabla_{Z}\left(\operatorname{Tr}\left(W_{22}\left(R+C Z C^{*}\right)\right)\right) \\
&=-C^{*}\left(R+C Z C^{*}\right)^{-1} C+W_{11}-A^{*} W_{11} A \\
&-A^{*} W_{12} C-C^{*} W_{12}^{*} A-C^{*} W_{22} C .
\end{aligned}
$$

At the optimal point, using Eqs. (43) and (44)

$$
\begin{aligned}
\left.\nabla_{Z} L(k, Z, W)\right|_{k_{\mathrm{o}}, Z_{\mathrm{o}}, W_{\mathrm{o}}} \\
=-C^{*}\left(R_{\mathrm{o}}+C Z_{\mathrm{o}} C^{*}\right)^{-1} C+W_{11, \mathrm{o}} \\
-\left(A+C^{*} M_{\mathrm{o}}^{*}\right) W_{11, \mathrm{o}}(A+M C),
\end{aligned}
$$

where $\quad M_{\mathrm{o}}=-\left(S_{\mathrm{o}}+A Z_{\mathrm{o}} C^{*}\right)\left(R_{\mathrm{o}}+C Z_{\mathrm{o}} C^{*}\right)^{-1}$. The first-order optimality condition implies

$$
\begin{aligned}
& \left.\nabla_{Z} L(k, Z, W)\right|_{k_{\mathrm{o}}, Z_{\mathrm{o}}, W_{\mathrm{o}}}=0, \\
& -N_{\mathrm{o}} N_{\mathrm{o}}^{*}+W_{11, \mathrm{o}} \\
& \quad-\left(A+C^{*} M^{*}\right) W_{11, \mathrm{o}}(A+M C)=0,
\end{aligned}
$$

where $N_{\mathrm{o}}=C^{*}\left(R_{\mathrm{o}}+C Z_{\mathrm{o}} C^{*}\right)^{-1 / 2}$. Since Eq. (51) is a Lyapunov equation, $W_{11}>0$ if and only if the pair $\left(A+M_{\mathrm{o}} C, N_{\mathrm{o}}\right)$ is observable. As a result, since the observability of the $(A, C)$ pair implies observability of $\left(A+M_{\mathrm{o}} C, N_{\mathrm{o}}\right), W_{11}$ is positive, and therefore, Eq. (37) holds.

To conclude, in this section, we showed that the risk-sensitive or the minimum entropy FIR equalization problem can be posed as a finite-dimensional convex optimization problem (constrained analytic centering problem) and can therefore be efficiently solved using interior point algorithms. In the following section, we look at the design of 
mixed $H^{2} / H^{\infty}$ FIR equalizers. In the last section, we present some examples where we compare FIR filters with different optimality properties.

\section{Mixed $H^{2} / H^{\infty}$ FIR equalization}

Using convex optimization techniques, we can also find the solution to the mixed $H^{2} / H^{\infty}$ FIR equalization problem. This problem is equivalent to finding the $H^{\infty}$ optimal FIR equalizer which has the least $H^{2}$ norm. Since the set of $H^{\infty}$ optimal equalizers is a convex set, and the $H^{2}$ cost function is a convex function of the FIR equalizer coefficients, this is also a convex optimization problem. In fact, we can pose this problem as an SDP problem.

The following lemma [7], which provides the calculation of the $H^{2}$ norm as a function of the state space parameters, plays a central role in the SDP formulation of the mixed problem:

Lemma 3 ( $H^{\infty}$ norm bound). Given any transfer function $H(z)=C(z I-A)^{-1} B+D$ (not necessarily minimal), we have:

$\|H(z)\|_{2}^{2}=\frac{1}{2 \pi} \int_{-\pi}^{\pi} \operatorname{Tr}\left(H\left(\mathrm{e}^{\mathrm{j} \omega}\right) H^{*}\left(\mathrm{e}^{\mathrm{j} \omega}\right)\right) \mathrm{d} \omega<\beta^{2}$,

where $A$ is asymptotically stable, if and only if the following LMI in $X$ and $S$ is feasible:

$\left[\begin{array}{cc}A^{*} X A-X & A^{*} X B \\ B^{*} X A & B^{*} X B-I\end{array}\right]<0$,

$\left[\begin{array}{ccc}X & 0 & C^{*} \\ 0 & I & D^{*} \\ C & D & S\end{array}\right]>0$,

$\operatorname{Tr}(S)-\beta^{2}<0$,

$X>0$.

Therefore, minimizing $\beta^{2}$ under the above constraints together with $F(k, Z, \gamma) \geqslant 0$ would yield the mixed $H^{2} / H^{\infty}$ solution. Similar to the risk-sensitive FIR filter, the mixed $H^{2} / H^{\infty}$ solution has a desired average property which is the minimization of the mean-square error under some statistical assumptions about the disturbances.

\section{Examples of FIR $H^{\infty}$ equalizers}

Previously, we presented methods to obtain the FIR equalizer coefficients for the risk sensitive and the mixed $H^{2} / H^{\infty}$ criteria. In this section, we will illustrate the use of these methods for some sample channels.

The first setting that we consider is for the channel $H(z)=1+0.9 z^{-1}$ and the delay $d=0$. In order to obtain a geometrical picture for the set of equalizers, we consider the equalizers of length 2 . In Fig. 5, the bounded convex region, the spectrahedron [11], represents the set $K_{\gamma}, \gamma$-level $H^{\infty}$ optimal equalizers, for this setting. The contours for the risk-sensitive cost function are also drawn inside the spectrahedron. The point represented by "*” is the risk-sensitive equalizer which is obtained by the constrained analytic centering method described previously. The point marked by " + " is the $H^{2}$ solution which is apparently not $H^{\infty}$ optimal since it lies outside of the set of $H^{\infty}$-optimal equalizers. The point marked with "o" is the mixed $H^{2} / H^{\infty}$ solution. Since the $H^{2}$-optimal equalizer is not inside the spectrahedron of $H^{\infty}$ solutions, the mixed $H^{2} / H^{\infty}$ solution lies at the boundary where the $H^{2}$ cost function is minimum.

In Fig. 6, the error spectra for the equalizers of the above setting are shown. It is clear from this figure that, since both the risk-sensitive and mixed $H^{2} / H^{\infty}$ solutions are $H^{\infty}$ optimal, their error spectra have the same maximum value which is smaller than the maximum value of the $H^{2}$ equalizer's error spectrum. The total area under the error spectrum is clearly minimized by the $H^{2}$ solution as expected. Besides, the mixed $H^{2} / H^{\infty}$ solution has a smaller area under the error spectrum than in the risk-sensitive case; however, the error spectrum for the risk-sensitive equalizer is smaller than that of the mixed $H^{2} / H^{\infty}$ solution at most of the frequencies, especially around the frequencies where the error spectra for both have high values.

In Fig. 7, error spectra are shown for the case where

$$
\begin{aligned}
H(z)= & 1.0000-1.3963 z^{-1}+0.9638 z^{-2} \\
& -0.8713 z^{-3}+0.5593 z^{-4}-0.1389 z^{-5}
\end{aligned}
$$




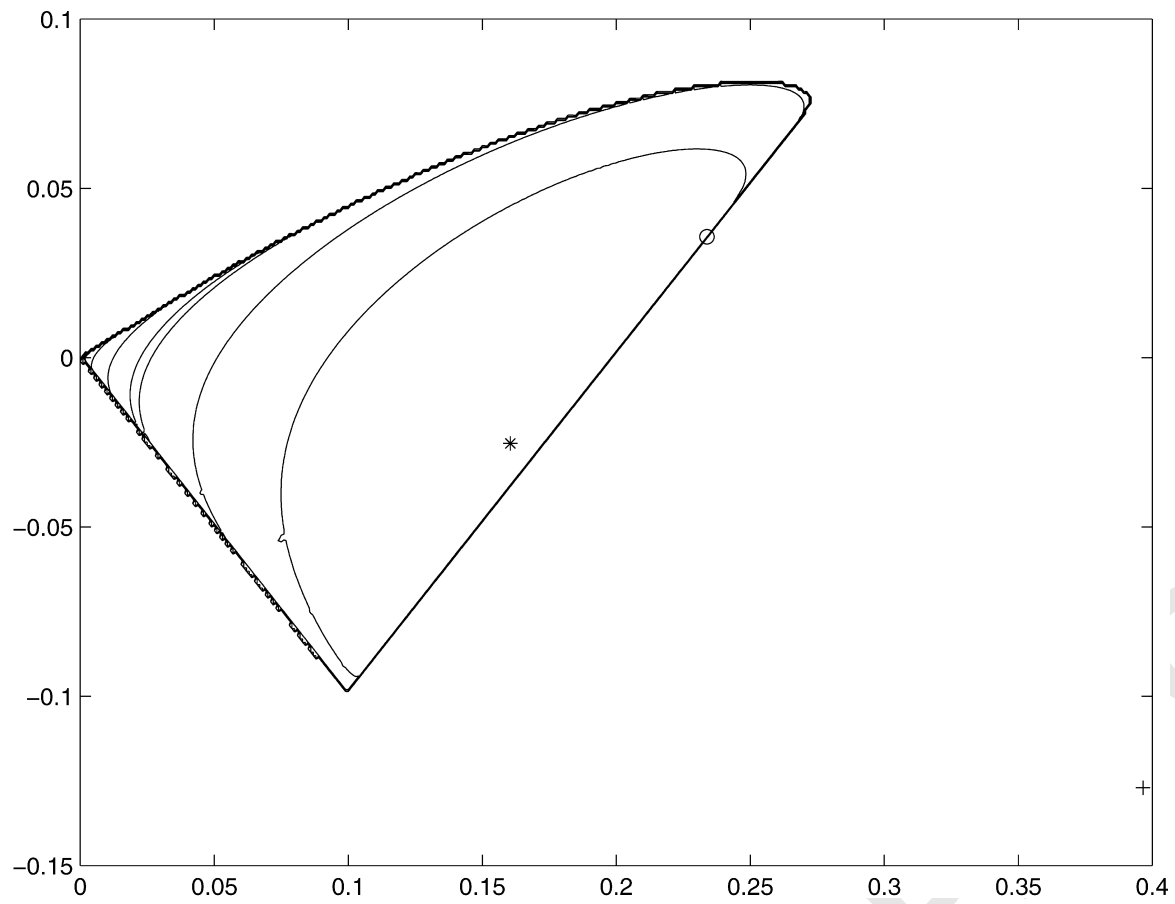

Fig. 5. $k_{0}$ vs. $k_{1}$. Two tap equalizers for $H(z)=1+0.9 z^{-1}$ and $d=0$ : “*”-Risk-sensitive solution, “+ "- $H^{2}$ solution, and "o"-Mixed $H^{2} / H^{\infty}$ solution.

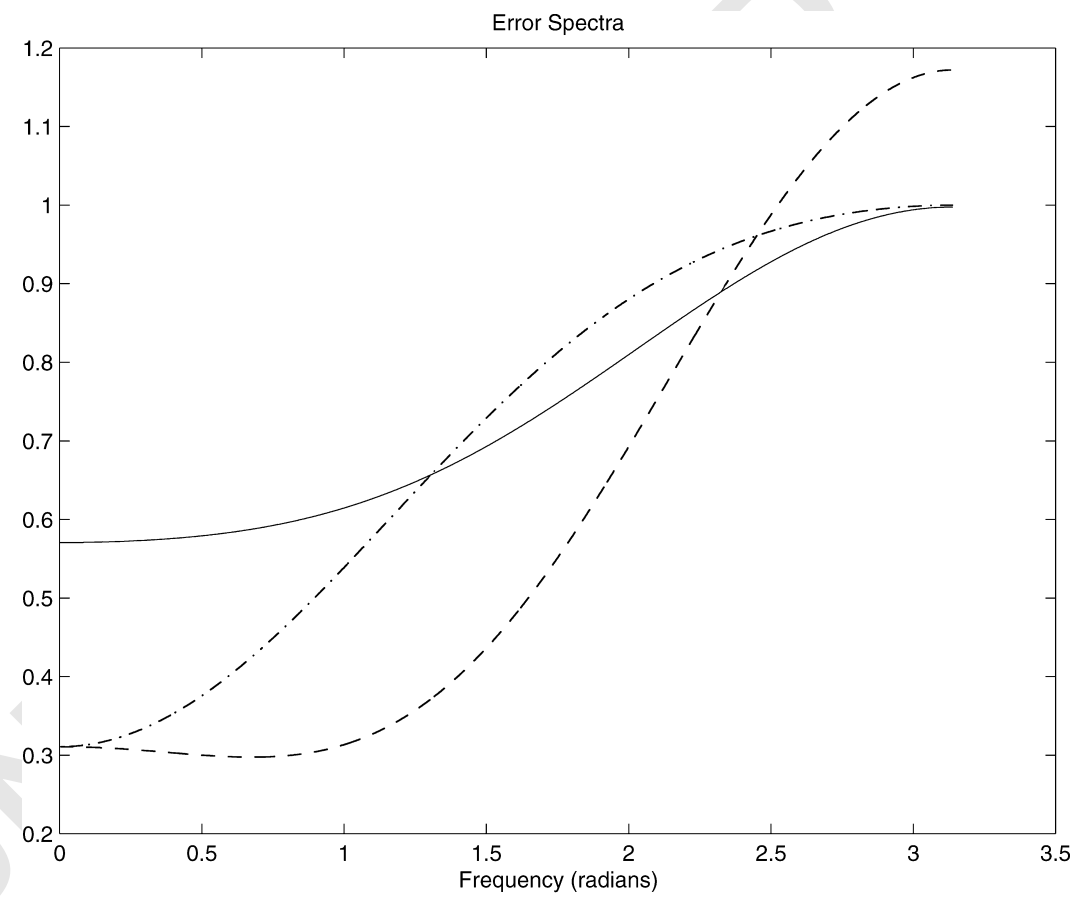

Fig. 6. The error spectra for two tap equalizers for $H(z)=1+0.9 z^{-1}$ and $d=0$ : (-), Risk-sensitive solution, $(----), H^{2}$ solution, and $(-\cdot-)$, mixed $H^{2} / H^{\infty}$ solution. 


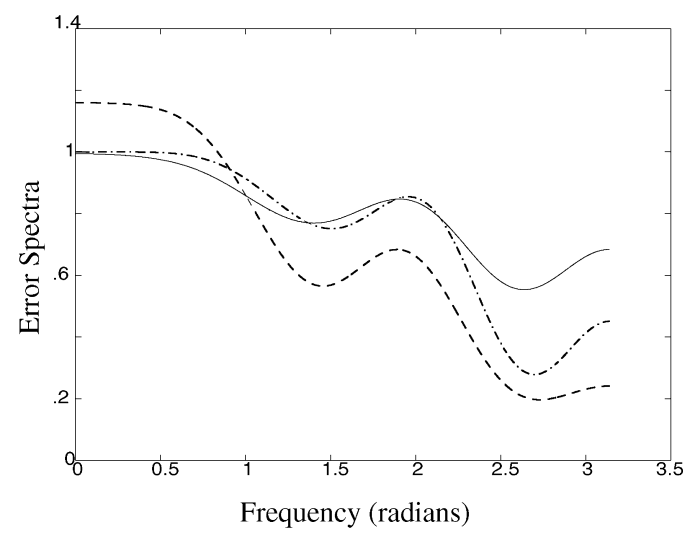

Fig. 7. The error spectra for two tap equalizers for $H(z)$ given in Eq. (56) and $d=0$ : (-), risk-sensitive solution, (- - - -), $H^{2}$ solution, and $(-\cdot-\cdot-)$, Mixed $H^{2} / H^{\infty}$ solution.

and $d=0$. The equalizer length is chosen to be equal to 4 in this case. Similar to previous example, the $H^{2}$-optimal FIR equalizer has the maximum value larger than the other $H^{\infty}$-optimal FIR equalizers although it minimizes the area under the error spectrum. For low frequencies, the risk-sensitive FIR equalizer has a smaller error spectrum than the mixed $\mathrm{H}^{2} / \mathrm{H}^{\infty}$ equalizer. However, for high frequencies, this condition is reversed and the mixed $H^{2} / H^{\infty}$ solution has considerably a smaller error spectrum.

\section{Conclusion}

In this article, we provided efficient convex optimization algorithms for $H^{\infty}$ FIR equalization. At the beginning, we posed the achievable performance level as an SDP problem. As $H^{\infty}$ criterion provides a family of equalizers with the optimal level, we look at some members of this family with additional average optimality properties. In particular, we showed that risk-sensitive (minimum entropy) FIR equalizer coefficients can be obtained efficiently through use of constrained analytic centering problem. Furthermore, an SDP-based approach is formulated to yield the mixed $H^{2} / H^{\infty}$ equalizer, which is the $H^{\infty}$ equalizer with the minimum square error.

\section{Uncited References}

[12]

\section{References}

[1] F. Alizadeh, J. Haaberly, M. Overton, Complementarity and non-degeneracy in semidefinite programming, Math. Program. (1995) submitted for publication.

[2] S. Boyd, L. Vandenberghe, Convex Optimization Course Reader, Stanford University, 1997.

[3] A.T. Erdogan, B. Hassibi, T. Kailath, Linear $H^{\infty}$ equalization of communication channels, IEEE Trans. Signal Process. September (2000) to appear.

[4] A.T. Erdogan, B. Hassibi, T. Kailath, Decision feedback equalization from an $H^{\infty}$ perspective, IEEE Trans. Signal Process., submitted for publication.

[5] K. Glover, J. Doyle, State-space formulae for all stabilizing controllers that satisfy an $H^{\infty}$ norm bound and relations to risk sensitivity, System Control Lett. 11 (1988) 167-172.

[6] B. Hassibi, A. Sayed, T. Kailath, Indefinite Quadratic Estimation and Control: A Unified Approach to $H^{2}$ and $H^{\infty}$ Theories, SIAM Studies in Applied Mathematics, New York, 1998.

[7] H. Hindi, B. Hassibi, S. Boyd, Multiobjective $H^{2} / H^{\infty}$ optimal control via finite dimensional $Q$-parametrization and linear matrix inequalities, Proceedings of American Control Conferences, vol. 5, 1998, pp. 3244-3249.

[8] P. Lancaster, L. Rodman, Algebraic Riccati Equations, Oxford Science Publications, Oxford, 1995.

[9] S.C. Peng, An equalizerd design for nonminimum phase channel via a two block $H^{\infty}$ optimization technique, Signal Process. 51 (1996) 1-18.

[10] J.G. Proakis, Digital Communications, McGraw-Hill, New York, 1995.

[11] M. Ramana, A. Goldman, Some geometric results in semidefinite programming, RUTCOR Research Report, 1994.

[12] A. Stoorvogel, A. Saberi, The discrete algebraic riccati equation and linear matrix inequality, Proceedings of IEEE Conferences on Decision and Control, 1994.

[13] L. Vandenberghe, S. Boyd, Semidefinite programming, SIAM Rev. 88 (1996) 49-95.

[14] P. Whittle, A risk-sensitive maximum principle, Systems Control Lett. 15 (1989).

[15] S. Wu, Max-det programming with applications in magnitude filter design, Ph.D. Thesis, Stanford University, 1997. 